\title{
Commentary Computer says 2.5 litres - how best to incorporate intelligent software into clinical decision making in the intensive care unit? Katie Lane and Owen Boyd
}

Department of Critical Care Medicine, Royal Sussex County Hospital, Eastern Road, Brighton, BN2 5BE, UK

Corresponding author: Owen Boyd, owen.boyd@bsuh.nhs.uk

Published: 23 January 2009

This article is online at http://ccforum.com/content/13/1/111

(c) 2009 BioMed Central Ltd

See related research by Celi et al., http://ccforum.com/content/12/6/R151
Critical Care 2009, 13:111 (doi:10.1186/cc7156)

\section{Bayesian network generated from observed data alone}

What will be the role of the intensivist when computer-assisted decision support reaches maturity? Celi's group reports that Bayesian theory can predict a patient's fluid requirement on day 2 in $78 \%$ of cases, based on data collected on day 1 and the known associations between those data, based on observations in previous patients in their unit. There are both advantages and limitations to the Bayesian approach, and this test study identifies areas for improvement in future models. Although such models have the potential to improve diagnostic and therapeutic accuracy, they must be introduced judiciously and locally to maximize their effect on patient outcome. Efficacy is thus far undetermined, and these novel approaches to patient management raise new challenges, not least medicolegal ones.

\section{Introduction}

Does the computer-driven prediction of fluid requirement spell the beginning of the end for the intensivist's daily management of the critically ill patient? Does it instead represent a useful adjunct to fluid balance assessment in the critically ill?

In the previous edition of Critical Care, Celi and coworkers [1] describe an artificial intelligence tool that can predict the quantity of fluid a critically ill patient will require on their second day of intensive care. From a database of 3,014 patients receiving inotropic support, Celi and colleagues constructed a Bayesian network [see Additional data file 1] [2]. The outcome variable was fluid requirement on day 2 , and input variables (nodes) were data collected within the first 24 hours of the intensive care unit stay, such as fluid intake and output, heart rate and blood pressure. When the model derived from the training data set was applied to a test set, it predicted the correct quartile of fluid requirement in $77.8 \%$ cases.
This pilot study differs from most previous attempts at computerized decision making in two respects. First, it addresses a therapeutic rather than diagnostic question. Second, the very predictive system itself has been generated from data unique to that patient, rather than an algorithm or guideline integrating opinion and best available medical evidence. The Bayesian system is a type of decision support system, as are logistic regression models and neural networks. The Bayesian approach has several practical advantages applicable to critical care, such as its ability to deal with uncertainties, for instance missed readings.

It also offers an intriguing means of circumventing the difficulties of applying the evidence from (often insufficiently powered) randomized controlled trials to the individual patient, by allowing patients to generate, to some extent, their own personal data set. The inductivist Sir Francis Bacon wrote, 'If we begin with certainties we shall end in doubts, but if we begin with doubts, and are patient with them, we shall end in certainties' [3]. A leitmotif in the use of computerized decision models is the difficulty in applying statistical tools to problems where the true answer is in doubt and may indeed be subject to large variations in clinical practice. This not only hampers the generation of an accurate model, but it also precludes accurate comparison of the model's efficacy with current clinical practice.

One approach to managing doubt is exemplified by Bayesian diagnosis of ventilator-associated pneumonia [4,5]. The algorithm correctly identified ventilator-associated pneumonia, with a positive predictive value of $87 \%$, and was concluded to be a useful adjunct to clinical acumen. In this case, the 
Bayesian network's original probability assessments were based either on the subjective assessments of two expert clinicians, or on scientific literature, and then updated using machine learning techniques. By contrast, the outcome in the retrospective analysis conducted by Celi and coworkers [1] was assumed to be the amount of fluid administered on the second day. Hence, the model's ability to predict fluid requirement can only be as good as the initial clinical assessment. This study was conducted in a single centre, and so the model in fact predicts the decisions of the clinicians who looked after the patients in the first place.

Of course, the incorporation of expert clinician fluid assessment into the original Bayesian network may have improved its accuracy. This would be an interesting addition to future work, as would comparing physician and computer model predictions. It will be important to include patient outcome measures as end-points in such a study, to provide some evidence of the effect of computer-aided decision systems on patient outcome [6].

\section{Study technicalities that could be improved}

The diversity of conditions studied was identified by the authors as a source of inaccuracy, but this could be rectified in forthcoming prospective studies. Furthermore, a more clinically relevant end-point could have been used. For example, in sepsis and postoperative patients, studies of early goal-directed therapy stress the importance of optimal fluid filling during the first 6 to 12 hours [7]. A more relevant data set might have been early fluid requirement during the first 12 to 24 hours after admission.

Other data might have improved the accuracy of this model. Presumably, the data chosen reflect the departments involved and the need for relative simplicity in this test study. For example, it is surprising that central venous pressure, arterial systolic pressure variation or pulmonary artery occlusion pressure, and the response of these variables to fluid challenge do not feature on the algorithm $[8,9]$. The utility of clinical parameters such as peripheral oedema and capillary refill to improve the accuracy with which fluid balance can be predicted remains undetermined. Addressing differences in data from different intensive care departments, both in terms of availability and interpretation, will be a major challenge for the wider use of Bayesian algorithms. Currently, there is good evidence that such models are most successfully applied when they are locally generated $[6,10]$.

\section{Conclusion}

There may be far-reaching implications of the incorporation of intelligence systems into clinical care. Medicolegal challenges to nonadherence to the computer-derived protocol may be difficult to defend (and lawyers have a good understanding of Bayesian theory!), and departures from recommendations and protocols will have to be carefully documented. Furthermore, we suspect that there will also be a fear of diverging from conclusions suggested by 'intelligent software', particularly where there is already doubt and difference in clinical opinion. In addition, there is a tendency to concur with a definite-looking computer-generated answer rather than trust one's own intuition.

There are many aspects of the management of the critically ill to which similar decision tools could be applied, such as antibiotic therapy, inotropic dosing and weaning from respiratory support. The future integration of these tools with molecular, laboratory and radiological data, as well as pathophysiology and associated co-morbidity, may well increase their power. Consideration of such factors as diagnosis and detection of complications and selection of therapeutic options is crucial in the management of the critically ill. Therefore, a place will remain for intuition, and for the human eyes, hands and brain at the critical care bedside.

\section{Additional data file}

The following Additional data file for this article is available online: Additional data file 1 is a Word document providing a definition of terms in a Bayesian network. See http://ccforum. com/content/supplementary/cc7156-s1.doc

\section{Competing interests}

The authors declare that they have no competing interests.

\section{References}

1. Celi LA, Hinske, LC, Alterovitz, G, Szolovits P: An artificial intelligence tool to predict fluid requirement in the intensive care unit: a proof-of-concept study. Crit Care 2008, 12:R151.

2. Schurink CAM, Visscher S, Lucas PJF, van Leeuwen HJ, Buskens E, Hoff RJ, Hoepelman AIM, Bonten MJM: A Bayesian decision support system for diagnosing ventilator-associated pneumonia. Intensive Care Med 2007, 33:1379-1386.

3. Bacon F. De Augmentis Scientarium, Book 1. 1605.

4. Schurink CAM, Lucas PJF, Hoepelman AIM, Bonten MJM: Computer-assisted decision support system for diagnosing ventilator-assisted pneumonia. Lancet Infect Dis 2005, 5:305-312.

5. Garg AX, Adhikari NKJ, McDonald H: Effects of computerised clinical support systems on practitioner performance and patient outcomes: a systematic review. JAMA 2005, 293: 1223-1238.

6. Dellinger RP, Levy MM, Carlet JM, Bion J, Parker MM, Jaeschke R, Reinhart K, Angus DC, Brun-Buisson C, Beale R, Calandra T, Dhainaut JF, Gerlach H, Harvey M, Marini JJ, Marshall J, Ranieri M, Ramsay G, Sevransky J, Thompson BT, Townsend S, Vender JS, Zimmerman JL, Vincent JL: Surviving Sepsis Campaign: international guidelines for management of severe sepsis and septic shock. Crit Care Med 2008, 36:296-327

7. Ornstein E, Eidelman LA, Drenger B, Elami A, Pizov R: Systolic pressure variation predicts the response to acute blood loss. J Clin Anesth 1998, 10:137-140.

8. Tavernier B, Makhotine O, Lebuffe G, Dupont J, Scherpereel P: Systolic pressure variation as a guide to fluid therapy in patient with sepsis-induced hypotension. Anesthesiology 1998, 89:1313-1321.

9. Chaudhry B, Wang B, Wu S. Maglione M, Mojica W, Roth E, Morton SC, Shekelle PG: Systematic review: Impact of health information technology on quality, efficiency, and costs of medical care. Ann Intern Med 2006, 144:742-752.

10. Goodman SN: Toward evidence-based medical statistics 2: The Bayes Factor. Ann Intern Med 1999, 130:1005-1013. 